\title{
Analysis of clinical factors and PDGFR- $\beta$ in predicting prognosis of patients with clival chordoma
}

\author{
Yixuan Zhai, MD,1 Jiwei Bai, MD, ${ }^{2}$ Shuai Wang, MD, ${ }^{1}$ Hua Gao, MD, ${ }^{1}$ Mingxuan Li, MD, \\ Chuzhong Li, MD, ${ }^{1}$ Songbai Gui, MD, PhD, ${ }^{2}$ and Yazhuo Zhang, MD, PhD' \\ 'Beijing Neurosurgical Institute, Capital Medical University; and 'Department of Neurosurgery, Beijing Tiantan Hospital, Capital \\ Medical University, Beijing, China
}

\begin{abstract}
OBJECTIVE In this study, the authors' aim was to research clinical features and prognostic factors in patients harboring clival chordomas and explore the relationship between platelet-derived growth factor receptor- $\beta$ (PDGFR- $\beta$ ) expression and tumor invasion and prognosis of clival chordoma.

METHODS A total of 242 patients were retrospectively analyzed. Clinical information, including extent of resection, AlMefty classification, postoperative complications, and postoperative radiotherapy, was reviewed. Kaplan-Meier analysis was used to estimate survival time. Immunohistochemical analysis, quantitative reverse transcription polymerase chain reaction, and Western blotting were used to measure the expression level of proteins or mRNA. Transwell assaying was performed to measure the invasive ability of the tumor cells.
\end{abstract}

RESULTS According to the Al-Mefty classification, there were 37, 112, and 93 type I, II, and III tumors, respectively. Gross-total resection (GTR) was achieved in 86 cases (35.5\%), subtotal resection (STR) in 63 cases (26.0\%), and partial resection (PR) in 93 cases (38.4\%). The 5-year progression-free survival (PFS) and overall survival (OS) rates in the GTR group were significantly higher than those in the non-total resection (NTR; i.e., STR and PR) group $(p<0.001)$. The 5 -year PFS and OS rates for patients with type I tumors were significantly higher than those for patients harboring types II and III tumors $(p<0.001)$. In the NTR group, the median PFS and OS of patients with lower PDGFR- $\beta$ expression were significantly longer than those of patients with higher PDGFR- $\beta$ expression. Reduction of PDGFR- $\beta$ suppressed the invasion ability of cells in vitro. In addition, reduction of PDGFR- $\beta$ can obviously downregulate the expression levels of mammalian target of rapamycin (mTOR) or phospho-mTOR.

CONCLUSIONS Extent of resection, Al-Mefty classification, primary tumor, postoperative radiotherapy, and PDGFR- $\beta$ expression level are valuable prognostic factors in patients with clival chordomas. PDGFR- $\beta$ could regulate invasion through the mTOR pathway in clival chordoma cells.

https://thejns.org/doi/abs/10.3171/2017.6.JNS17562

KEY WORDS clival chordomas; degree of resection; classification; radiotherapy; PDGFR- $\beta$; invasion; prognosis; oncology

$\mathrm{C}$ HORDOMAS are rare, slow-growing bone tumors that account for $1 \%-4 \%$ of all malignant bone tumors. ${ }^{19}$ The tumors are often located in the axial skeleton: $50 \%$ in the sacrococcygeal region, $35 \%$ in the clival region, and $15 \%$ in the vertebrae. ${ }^{32}$ As chordoma is resistant to conventional chemotherapy and radiotherapy, radical resection remains the surgeon's first choice for treatment. ${ }^{22,23}$ However, achieving gross-total resection
(GTR) is a great challenge due to the proximity of the lesion to vital structures..$^{14}$ Moreover, the prognosis remains unsatisfactory. Therefore, better understanding of a chordoma's biology and mechanism of invasion is needed to improve patient prognosis and ensure that patients receive optimal treatment.

Platelet-derived growth factor receptor- $\beta$ (PDGFR- $\beta$ ) is a $124-\mathrm{kD}$ protein kinase belonging to the receptor ty-

ABBREVIATIONS GTR = gross-total resection; KPS = Karnofsky Performance Scale; $m$ TOR = mammalian target of rapamycin; NTR = non-total resection; OS = overall survival; PDGFR- $\beta=$ platelet-derived growth factor receptor $-\beta ; \mathrm{PFS}=$ progression-free survival; $\mathrm{PR}=$ partial resection; $\operatorname{siRNA}=$ small interfering RNA; $\mathrm{STR}=$ subtotal resection; TMA = tissue microarray.

SUBMITTED March 5, 2017. ACCEPTED June 5, 2017

INCLUDE WHEN CITING Published online January 5, 2018; DOI: 10.3171/2017.6.JNS17562. 
rosine kinase family. Stimulation of PDGFR- $\beta$ leads to activation of intracellular signaling pathways that promote cell migration, invasion, cell growth, and survival. ${ }^{10,20}$ The PDGFR signaling pathway contributes to the aggressive behavior of epithelial tumor types, such as colorectal, breast, liver, and pancreas carcinomas. .11,18,25 $^{2}$ Moreover, high PDGFR- $\beta$ expression correlates with poor prognosis in these tumor types. ${ }^{11,18}$

In this article, we discuss the clinical features and prognostic factors in patients harboring clival chordomas and explore the relationship between PDGFR- $\beta$ expression and tumor invasion and prognosis of clival chordoma.

\section{Methods \\ Patient Selection}

The files of the Department of Neurosurgery, Beijing Tiantan Hospital, Capital Medical University, were searched for patients who underwent clival chordoma treatment between March 2004 and September 2014. Patients were selected according to the following inclusion criteria: 1) chordoma diagnosis confirmed by pathological report, 2) complete medical records, and 3) positive immunostaining of Brachyury and cytokeratin. Excluding criteria were as follows: 1) insufficient size of the tumor specimen for building a tissue microarray (TMA), and 2) incomplete medical records.

\section{Clinical Information Collection}

The clinical information was collected by 2 members of our team (Y.Z. and J.B.). Two experienced neurosurgeons (C.L. and J.B.) reviewed the images to determine residual, recurrent, or progressive disease. When there were conflicting opinions between these team members, S.G. was consulted. All patients signed the informed consent agreement, and our study protocol was approved by the ethics committee of Beijing Tiantan Hospital.

Postoperative images were compared with preoperative images to determine the extent of tumor resection. Patients were divided into 3 categories: total resection (no residual tumor on the postoperative images), subtotal resection (STR; $\geq 95 \%$ resection), and partial resection (PR; $<95 \%$ resection). ${ }^{9}$

According to the Al-Mefty classification, ${ }^{1}$ we divided the tumors into 3 types. Type I tumors were restricted to 1 compartment of the skull base. Type II tumors extended to 2 or more contiguous areas, and radical resection could be achieved using a single approach. Type III tumors extended to several contiguous areas, and radical resection could not be achieved using a single approach.

\section{Immunohistochemical Staining and Analysis}

Formalin-fixed, paraffin-embedded chordoma specimens were used to construct the TMA using a Tissue Array MiniCore 3 (ALPHELYS SAS). Four-micron-thick sections were cut from each TMA using a rotary microtome (RM2135, Leica). Immunohistochemical staining was performed using a Leica BOND III automated system, and protocol $\mathrm{F}$ was chosen. The sections were incubated with the PDGFR- $\beta$ antibody (dilution 1:100, Cell Signaling Technology). Immunostained TMA sections were scanned using a Leica Aperio AT2 scanner (at 400x magnification) and analyzed using a Leica Aperio ImageScope version 12.3.0.5056. The membrane version 9 algorithm was chosen for antibody positivity in membranes. An HScore of each case was established using the following formula: HScore $=1 \times$ (percentage of light staining $)+$ $2 \times$ (percentage of moderate staining) $+3 \times$ (percentage of strong staining). Thus, the HScore ranges from 0 to $300 . .^{15}$

\section{Cell Culture and Cell Invasion Assays}

Chordoma cells (U-CH2), donated by the Chordoma Foundation, were cultured in Isocove's modified Dulbecco's medium (IMDM; Invitrogen)/1640-RPMI (Sigma-Aldrich) (4:1) culture medium supplemented with $10 \%$ fetal bovine serum in a humidified incubator at $37^{\circ} \mathrm{C}$ and $5 \%$ $\mathrm{CO}_{2}$. PDGFR- $\beta$ gene silencing in $\mathrm{U}-\mathrm{CH} 2$ cells was established by transfection of small interfering RNA (siRNA; OriGene) using Lipofectamine 3000 (Invitrogen).

Tumor cell invasion was measured by counting invasive cells that had been placed in $6.5-\mathrm{mm}$ transwell chambers, by means of Matrigel-coated polycarbonate filters (Corning). Transient transfected U-CH2 cells $\left(2.5 \times 10^{4}\right.$ cells) with PDGFR- $\beta$ siRNA or nonsilencing siRNA were added into the transwell chambers. After being cultured in an incubator for 24 hours, invasive cells adhering to the lower membrane were fixed in $4 \%$ paraformaldehyde and stained with hematoxylin. The invasive cells stained dark, and only the single stained cells were counted. The average number of invasive cells was quantified by counting 5 random high-power fields under the microscope (Zeiss). ${ }^{17}$

\section{Western Blotting and Quantitative Reverse Transcription Polymerase Chain Reaction}

Transfected U-CH2 cells were lysed in nondenaturing lysis buffer (Applygen). For Western blotting, protein samples $(20 \mu \mathrm{g})$ were separated by $10 \%$ sodium dodecyl sulfate polyacrylamide gel electrophoresis and then transferred to polyvinylidene difluoride membranes. Different blots were incubated with antibodies against PDGFR- $\beta$ (1:1000, Cell Signaling Technology), phospho-PDGFR- $\beta$ antibody (1:1000, Abcam), mammalian target of rapamycin (mTOR) antibody (1:100, Cell Signaling Technology), or phospho-mTOR antibody (1:1000, Abcam) and GAPDH (1:5000, Topunive), followed by secondary antibodies tagged with horseradish peroxidase (Santa Cruz Biotechnology). Blots were visualized by enhanced chemiluminescence, and densitometry was performed with an imaging apparatus (Amersham Imager 600, GE). Analysis of GAPDH levels was used as a loading control.

The mRNA expression level of PDGFR- $\beta$ was measured using quantitative reverse transcription polymerase chain reaction. The first-strand cDNA was synthesized from total RNA (800 ng) using the SuperScript III FirstStrand Synthesis System (Invitrogen). Quantitative reverse transcription polymerase chain reaction was performed using an ABI 7500 Fast system (Applied Biosystems) with Power SYBR Green PCR Master Mix (Applied Biosystems). The primers were as follows: CGTCAAGATGCTT AAATCCACAGC (forward) and TGATGATATAGATG GGTCCTCCTTTG (reverse). 
TABLE 1. Summary of the patients

\begin{tabular}{|c|c|}
\hline Variable & Value \\
\hline \multicolumn{2}{|l|}{ Sex, $n$} \\
\hline Male & 133 \\
\hline Female & 109 \\
\hline Age in yrs, mean (range) & $41.29(8-78)$ \\
\hline KPS, mean (range) & $78.5(20-90)$ \\
\hline Duration of history in mos, mean (range) & $16.85(1-120)$ \\
\hline \multicolumn{2}{|l|}{ Chief complaints, $n$} \\
\hline Headache & 100 \\
\hline Diplopia & 77 \\
\hline Blurred vision & 70 \\
\hline Hemianopia & 55 \\
\hline Lower cranial nerve dysfunction & 35 \\
\hline Dizziness & 31 \\
\hline Vol in $\mathrm{cm}^{3}$, mean (range) & $32.7(1.7-556.6)$ \\
\hline \multicolumn{2}{|l|}{ Al-Mefty classification, $n$} \\
\hline Type I & 37 \\
\hline Type II & 112 \\
\hline Type III & 93 \\
\hline \multicolumn{2}{|l|}{ Pituitary gland involved, $\mathrm{n}$} \\
\hline Yes & 117 \\
\hline No & 125 \\
\hline \multicolumn{2}{|l|}{ Brainstem involved, $n$} \\
\hline Yes & 135 \\
\hline No & 107 \\
\hline \multicolumn{2}{|l|}{ Lower cranial nerves involved, $n$} \\
\hline Yes & 80 \\
\hline No & 162 \\
\hline \multicolumn{2}{|l|}{ Tumor calcification, $n$} \\
\hline Yes & 89 \\
\hline No & 153 \\
\hline Primary tumor, $n$ & 182 \\
\hline Recurrent tumor, $\mathrm{n}$ & 60 \\
\hline \multicolumn{2}{|l|}{ Op approach, $\mathrm{n}$} \\
\hline Craniotomy & 124 \\
\hline Endoscopic transsphenoidal approach & 108 \\
\hline Endoscopic transoral approach & 10 \\
\hline \multicolumn{2}{|l|}{ Extent of resection, $n$} \\
\hline TR & 86 \\
\hline STR & 63 \\
\hline PR & 93 \\
\hline
\end{tabular}

TR = total resection.

\section{Statistical Analysis}

Statistical analysis was performed using IBM SPSS software (version 22.0, IBM Corp.). The Kaplan-Meier method was used for survival analysis. Differences between groups were determined using the chi-square test and 2 independent samples t-test; $\mathrm{p}<0.05$ was defined as statistically significant. Graphs were constructed using Prism software (version 5.0, GraphPad).
TABLE 2. Association between extent of resection and Al-Mefty classification

\begin{tabular}{ccccc}
\hline \multirow{2}{*}{$\begin{array}{c}\text { Al-Mefty } \\
\text { Classification }\end{array}$} & \multicolumn{3}{c}{ No. of Patients (\%) } & \\
\cline { 2 - 4 } & TR & STR & PR & p Value \\
\hline Type I & $31(83.8)$ & $5(13.5)$ & $1(2.7)$ & \\
\cline { 1 - 3 } Type II & $54(48.2)$ & $36(32.2)$ & $22(19.6)$ & $<0.001$ \\
\cline { 1 - 3 } Type III & $1(1.1)$ & $22(23.6)$ & $70(75.3)$ & \\
\hline
\end{tabular}

\section{Results \\ Patient Summary}

A total of 242 patients (133 males and 109 females) who harbored 182 primary and 60 recurrent chordomas were included in the study. The mean patient age was 41.29 years (range $8-78$ years). The common complaints included headache (100 patients), diplopia (77 patients), blurred vision (70 patients), and hemianopia (55 patients). The Karnofsky Performance Scale (KPS) score ranged from 20 to 90 (mean KPS score 78.5). The tumor volume ranged from 1.7 to $556.6 \mathrm{~cm}^{3}$ (mean $32.7 \mathrm{~cm}^{3}$ ). According to the Al-Mefty classification, ${ }^{1}$ there were 37,112 , and 93 cases of type I, type II, and type III tumors, respectively. Intraoperative navigation and electrophysiological monitoring were used in 17 and 70 patients, respectively. Patient characteristics are given in Table 1.

The endoscopic transsphenoidal approach was used in 108 cases, while the endoscopic transoral approach was used in 10 cases and a craniotomy in 124 cases. Total resection was achieved in 86 cases (35.5\%), STR in 63 cases (26.0\%), and PR in 93 cases (38.4\%) (Table 1). The GTR rates for type I and type II tumors were significantly higher than that for type III tumors (Table 2). Postoperative radiation therapy was performed in 79 cases.

The most common postoperative complication was intracranial infection (18 cases), followed by new cranial nerve palsy (13 cases) and CSF leakage ( 8 cases). The postoperative complication rates in the GTR, STR, and PR groups were $27.5 \%, 25.0 \%$, and $21.5 \%$, respectively (p $=0.68)$. The postoperative complication rates of the endoscopic transsphenoidal approach and craniotomy were $13.7 \%$ and $36.7 \%$, respectively $(\mathrm{p}<0.001)$. In patients undergoing an endoscopic approach, GTR, STR, and PR were achieved in 39,32 , and 47 patients, respectively. In patients who underwent a craniotomy, GTR, STR, and PR were achieved in 47, 31, and 46 patients, respectively. There was no significant difference in extent of resection between different surgical approaches $(p=0.733)$.

\section{Clinical Factors Affecting Overall Survival and Progression-Free Survival}

The 5-year progression-free survival (PFS) rates for the GTR, STR, and PR groups were 50\%, $11 \%$, and $3 \%$, respectively ( $<<0.001)$, and the 5 -year overall survival (OS) rates in the GTR, STR, and PR groups were $86 \%, 68 \%$, and $32 \%$, respectively $(\mathrm{p}<0.001)$. The 5 -year PFS rates in patients with type I, II, and III tumors were $37 \%, 29 \%$, and $7 \%$, respectively $(\mathrm{p}<0.001)$, and the 5 -year OS rates in patients with type I, II, and III tumors were $77 \%, 70 \%$, and $44 \%$, respectively ( $<<0.001$; Fig. 1$)$. 

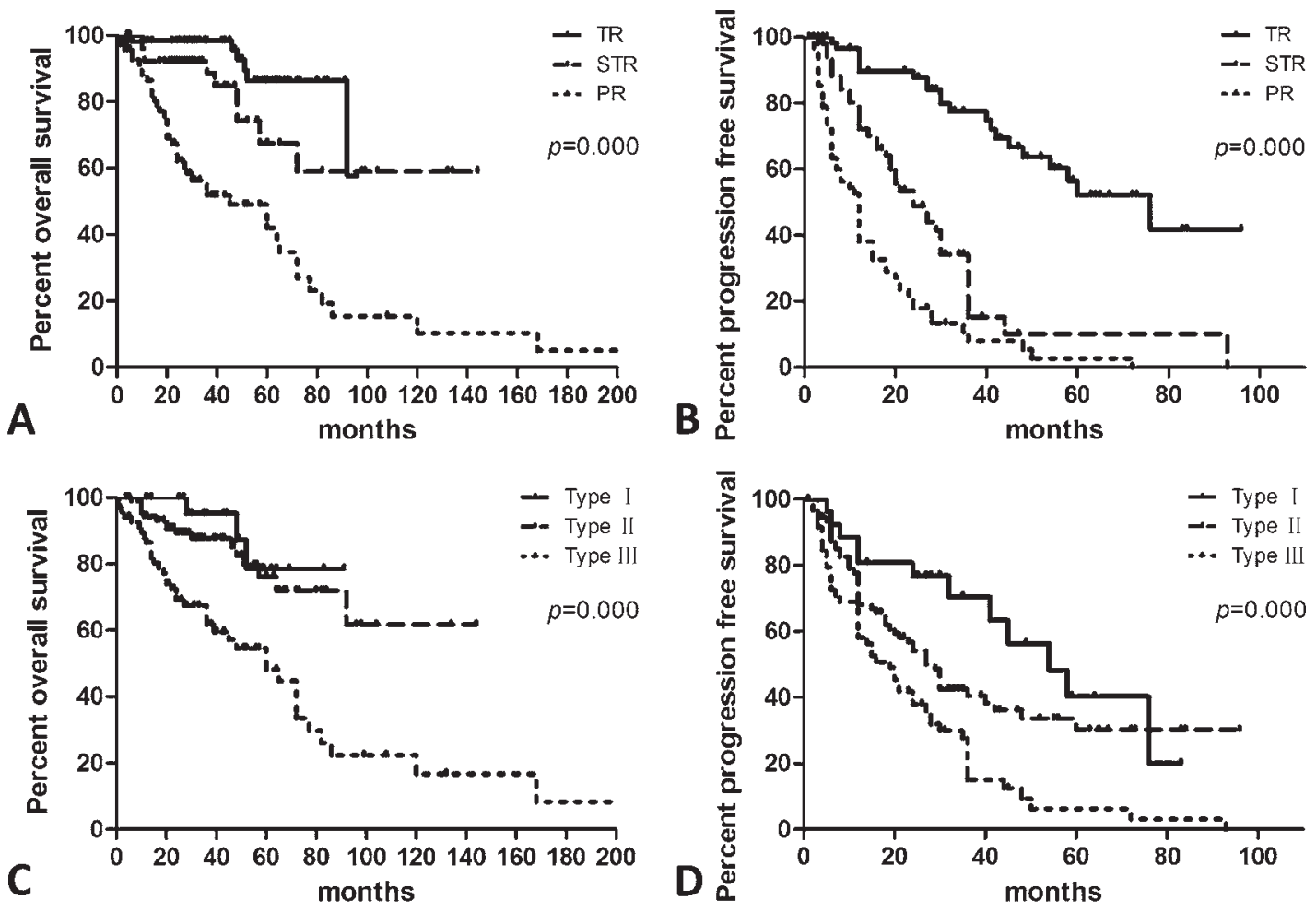

FIG. 1. Kaplan-Meier survival functions. A and B: GTR was associated with longer patient OS (A) and PFS (B). C and D: Type I tumors were associated with longer patient OS (C) and PFS (D).
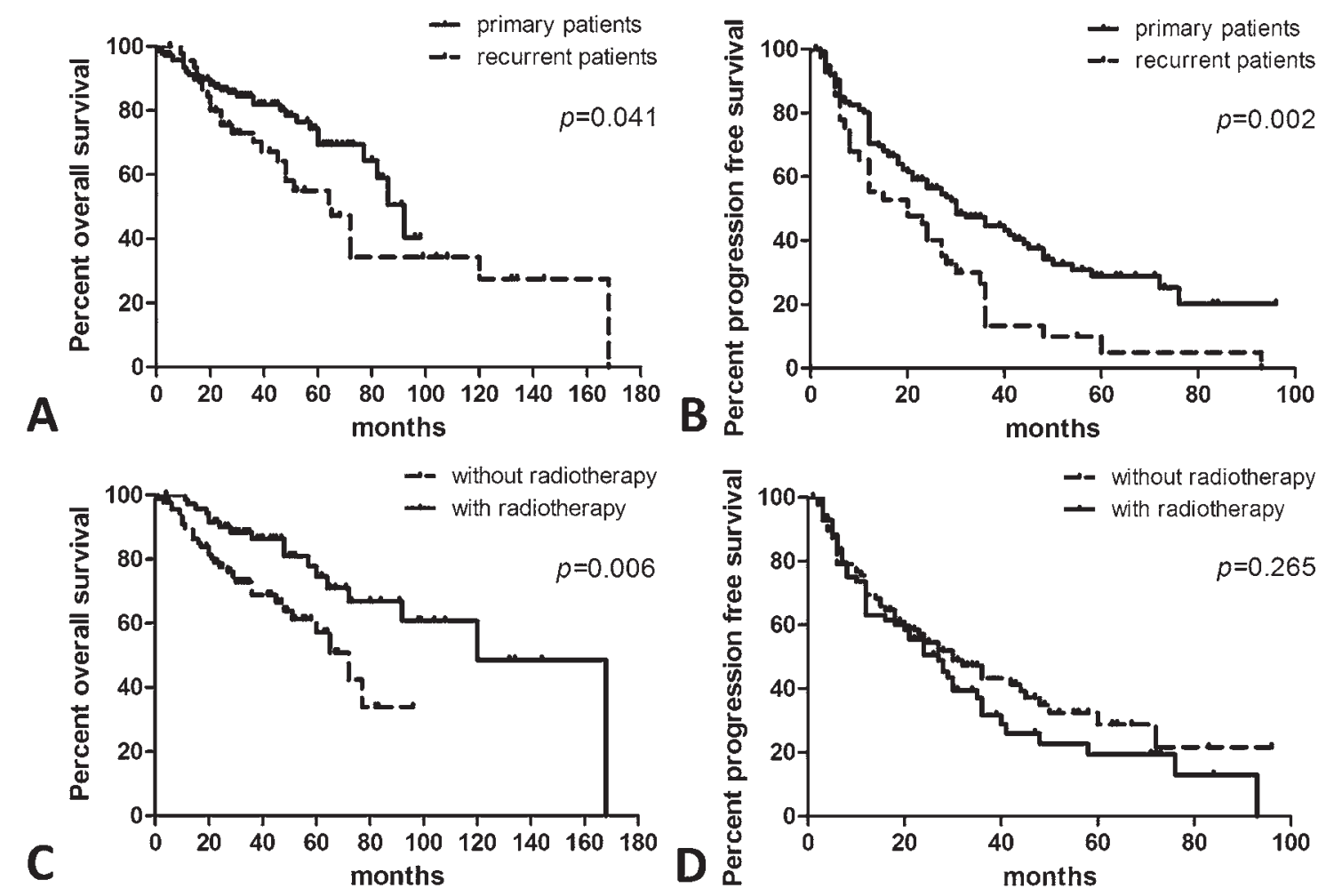

FIG. 2. Kaplan-Meier survival functions. A and B: The median OS (A) and PFS (B) for patients with primary tumors were significantly longer than those for patients with recurrence. C: The median OS of patients who underwent radiotherapy was significantly longer than that of patients who did not undergo radiotherapy. D: There was no difference in median PFS time between patients with radiotherapy and those without radiotherapy. 

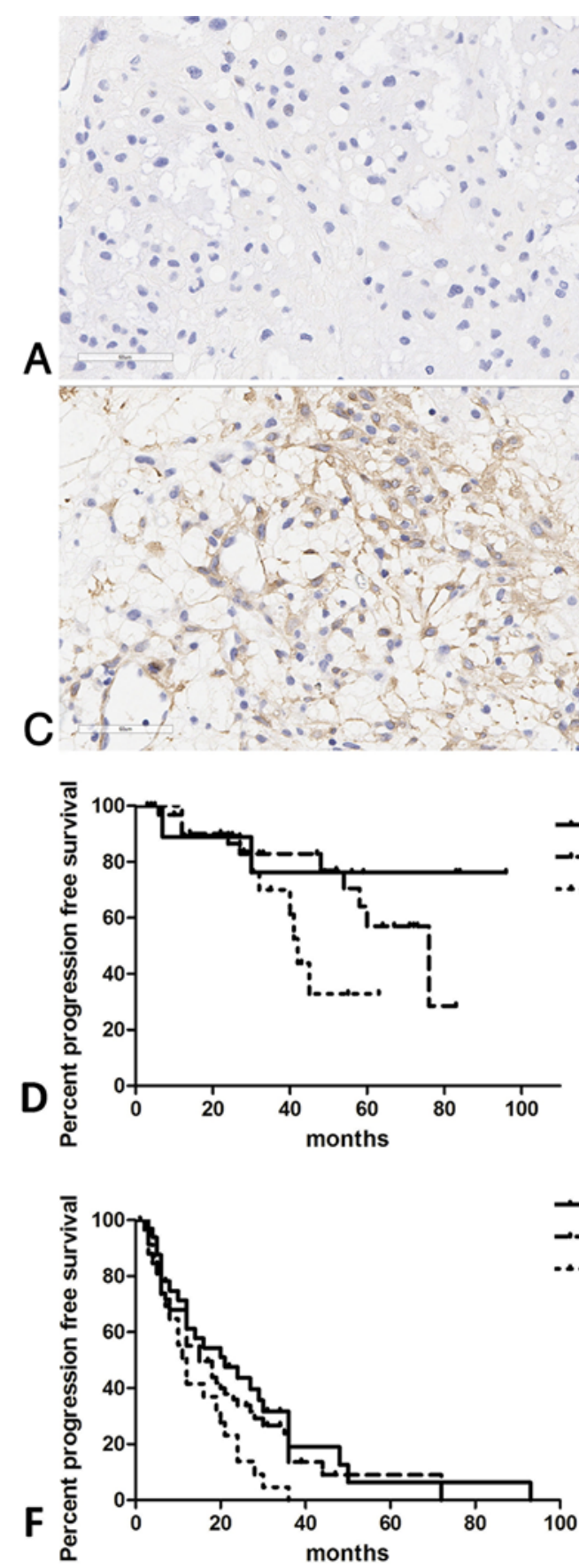

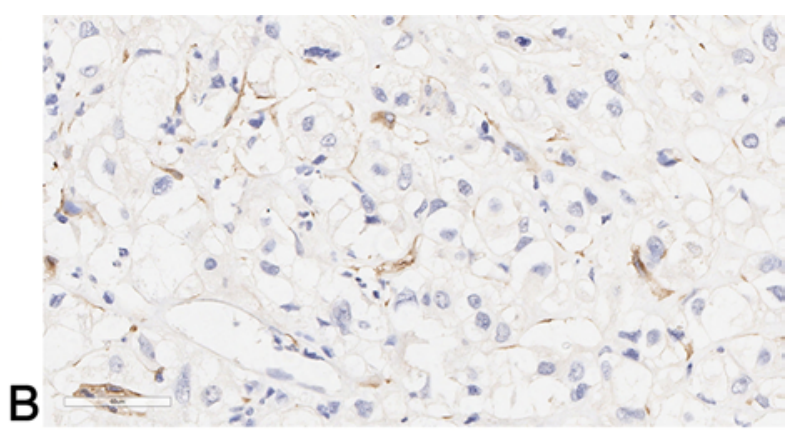

B

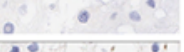

- Group A
$\ldots$ Group B
$\ldots$ Group C
$p=0.096$

- Group A
$\ldots$ Group B
$\ldots$ Group C
$\quad p=0.045$
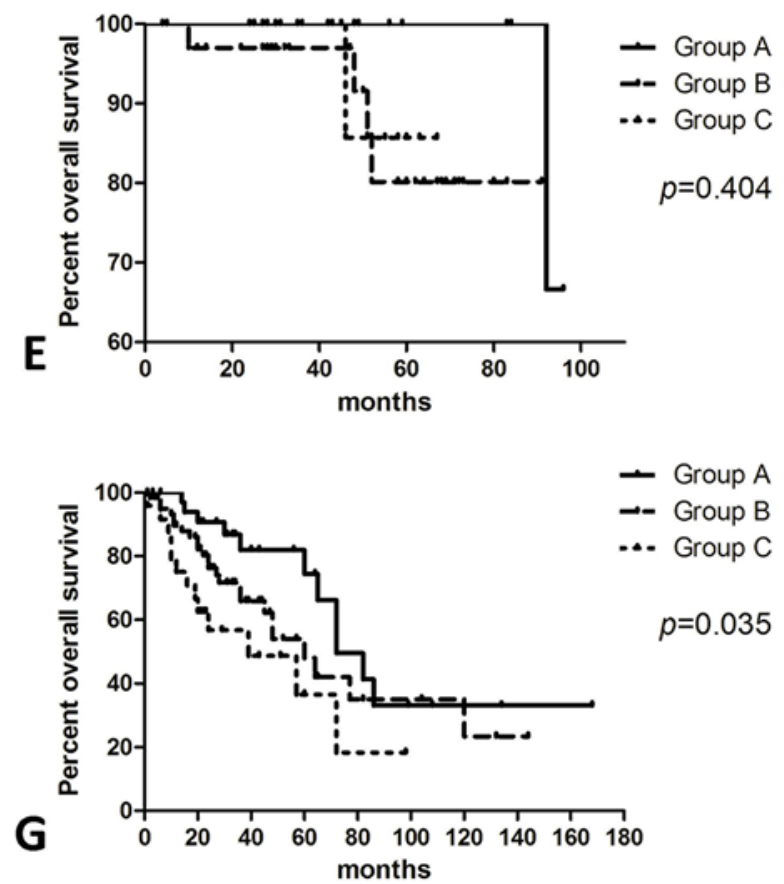

FIG. 3. A: Group A, HScore = 1.25. B: Group B, HScore = 88.15. C: Group C, HScore $=205.57$. D and E: In the GTR group, there was no significant difference between the 3 groups in terms of PFS (D) or OS (E). F and G: In the NTR group, the PFS (F) and OS $(\mathrm{G})$ for HScore group A were significantly longer than those of HScore groups B and C. Figure is available in color online only.

The median PFS of patients with primary tumors was 30 months, which was significantly longer than that of patients experiencing recurrence (20 months, $p=0.002)$. The median OS of primary patients was 86 months, which was significantly longer than that of patients with recurrence (65 months, $p=0.041$; Fig. 2). Among primary patients, GTR was achieved in 77 cases (42\%). Among patients with recurrence, GTR was achieved in only 9 cases (15\%), which was significantly lower than the number of cases of primary patients $(\mathrm{p}<0.001)$. There was no significant difference in complication rates between primary patients and those with recurrence ( $27 \%$ vs $20 \%, \mathrm{p}=0.284$ ).

Based on Kaplan-Meier analysis, the median OS of patients receiving radiotherapy was significantly longer than that of patients without radiotherapy (120 vs 72 months, $p$ $=0.006)$. There was no significant difference in median 


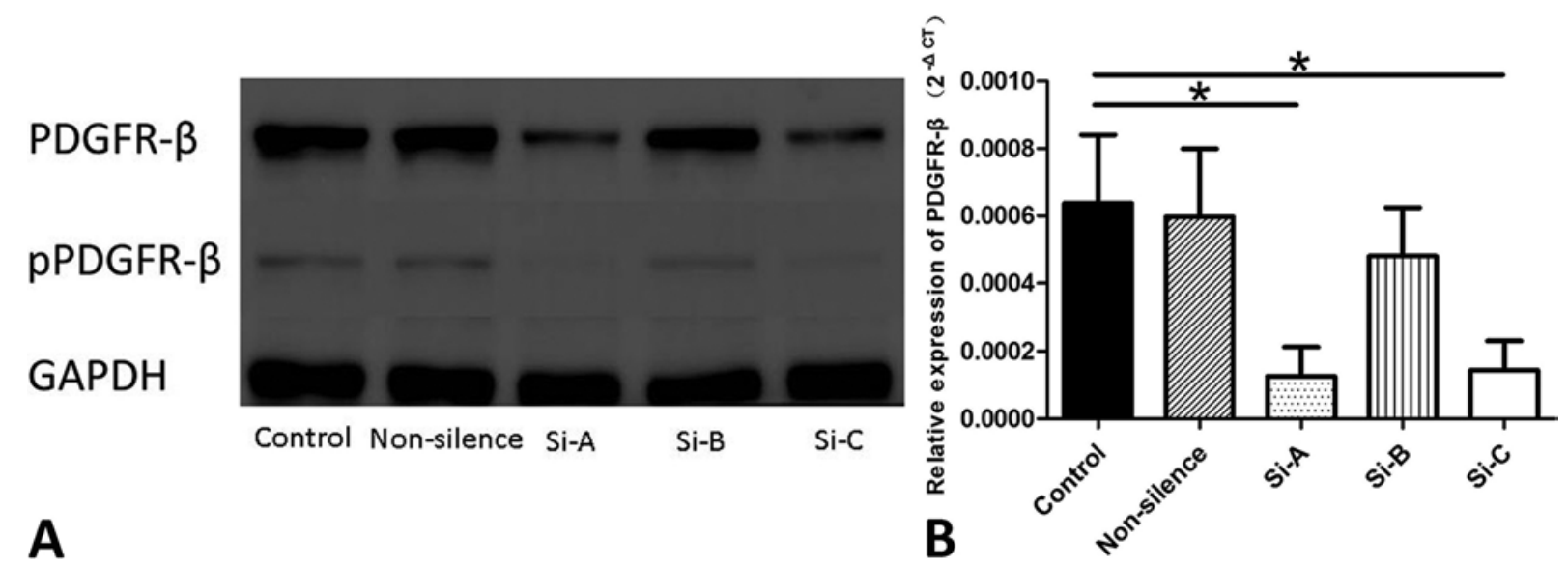

FIG. 4. A: Western blotting analysis showing that expression of PDGFR- $\beta$ and phospho-PDGFR- $\beta$ (pPDGFR- $\beta$ ) was significantly suppressed by Si-A and Si-C. B: Polymerase chain reaction analysis revealed that the PDGFR- $\beta$ mRNA level was significantly suppressed by Si-A and Si-C. ${ }^{*} p<0.05$.
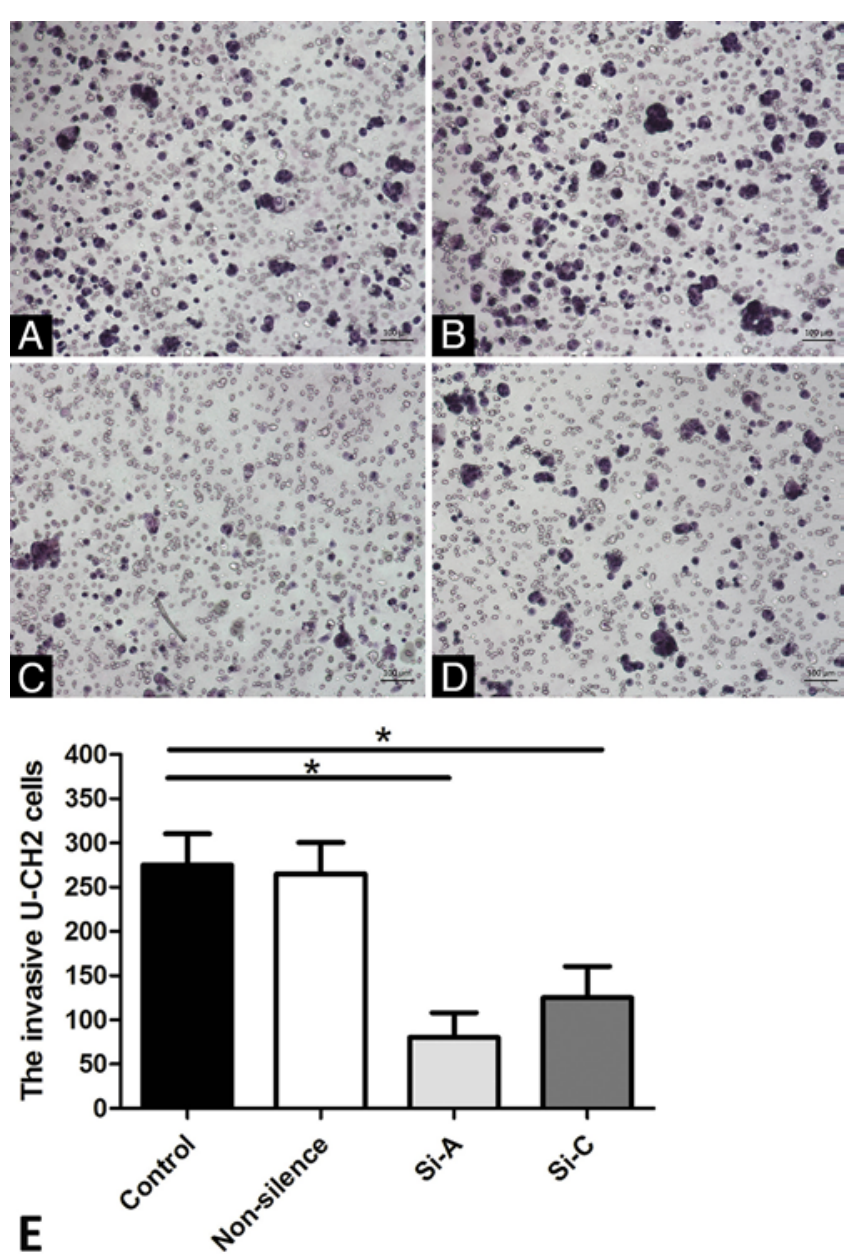

FIG. 5. Transwell assaying. The invasive cells in group Si-A and group Si-C were significantly decreased compared with those in the control group. A: Control. B: Nonsilence. C: Si-A. D: Si-C. The small round background circles are holes in the membranes of transwell chambers. The invasive cells stained dark; only the single stained cells were counted. E: The invasive cells in the Si-A and Si-C groups decreased significantly to $29 \%(p=0.026)$ and $44 \%(p=0.040)$ compared with those in the control group. ${ }^{*} p<0.05$. Figure is available in color online only.
PFS between patients receiving radiotherapy (27 months) and patients not receiving radiotherapy (30 months) ( $\mathrm{p}=$ 0.265; Fig. 2).

\section{Association Between PDGFR- $\beta$ Expression Level and Prognosis}

Based on immunohistochemical analysis, PDGFR- $\beta$ was located in the cell membranes in all chordoma cells with positive staining. The mean HScore was 70.31 (range 1.25-205.57). The median HScore was 63.8 (IQR 29.1102.9). Based on the 25th and 75th percentiles, patients were divided into 3 groups, as follows: group A, HScore < 29.1; group B, HScore = 29.1-102.9; and group C, HScore $>$ 102.9. In the GTR group, there was no significant difference between 3 groups in terms of PFS and OS. However, in the non-total resection (NTR) group, the median PFS times in HScore groups A, B, and C were 21, 15, and 12 months, respectively $(\mathrm{p}=0.045)$. The median OS times in groups A, B, and C were 72, 60, and 29 months, respectively ( $p=0.035$; Fig. 3 ). The mean HScores of PDGFR- $\beta$ in primary and recurrent chordomas were 72.41 and 68.21 , respectively, with no significant difference $(\mathrm{p}=0.671)$.

\section{Reduction of PDGFR- $\beta$ Expression Suppresses Invasion}

The impact of PDGFR- $\beta$ on tumor invasion was first measured in U-CH2 cells. Three different PDGFR- $\beta$ siRNAs were used, and the PDGFR- $\beta$ expression was reduced to $19.7 \%$ (Si-A), $75.2 \%(\mathrm{Si}-\mathrm{B})$, and $22.6 \%(\mathrm{Si}-\mathrm{C})$ at 48 hours after transfection (Fig. 4). Si-A and Si-C were selected in further assays because of their strong suppression ability. The transwell assay revealed that invasive cell counts significantly decreased when PDGFR- $\beta$ expression was suppressed (Fig. 5).

\section{PDGFR- $\beta$ Regulates Chordoma Invasion via the mTOR Pathway}

The mTOR pathway plays a crucial role in the regulation of cell motility ${ }^{16}$ and invasion. ${ }^{2}$ Western blot analysis detected that the expression levels of phospho-PDGFR- $\beta$, mTOR, and phospho-mTOR decreased significantly after suppression of PDGFR- $\beta$ expression (Figs. 4 and 6). 


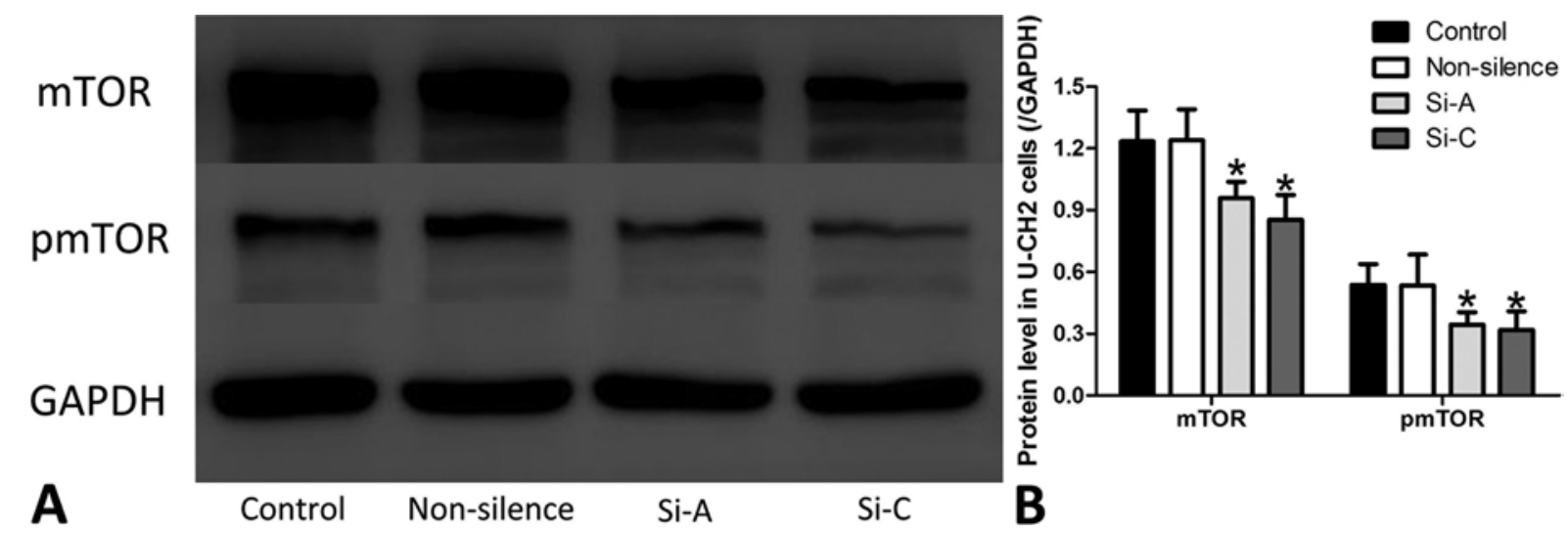

FIG. 6. PDGFR- $\beta$ silencing regulates the mTOR pathway. A: Western blot analysis of mTOR and phospho-mTOR (pmTOR) in $\mathrm{U}-\mathrm{CH} 2$ cells. B: The Western blot analysis reveals a significant reduction of mTOR and phospho-mTOR in the Si-A and Si-C groups compared with those in the control group. ${ }^{*} p<0.05$.

\section{Discussion}

In line with previous studies, ${ }^{13}$ we have shown that clival chordoma is more prevalent in male patients and mainly involves patients who are approximately 40 years old. Many studies ${ }^{22,23,28}$ have reported that radical resection can improve a patient's prognosis. With the assistance of modern equipment, such as intraoperative navigation, intraoperative laser, and transcranial Doppler ultrasonography, it is possible to locate and protect important structures in advance, which obviously decreases the complication rates. In our study, we found that GTR and STR can significantly improve prognosis, and there was no correlation between extent of resection and postoperative complications. Thus, radical resection with protection of important structures is the goal of the surgery. An endoscopic approach and craniotomy are the most common approaches. An endoscopic approach has the advantages of broader vision and lower postoperative complication rates. If the largest portion of the chordoma is located in the midline region, an endoscopic approach may be more convenient than a craniotomy. Moreover, we found that there is no significant difference in extent of resection between an endoscopic approach and a craniotomy. Thus, we consider that an endoscopic approach is preferable to craniotomy in chordoma patients. If the largest portion of the chordoma is not located in the midline, or the tumor penetrates the dura mater and severely compresses the brainstem or nerves, a craniotomy might be considered. It is important to note that in our study, the endoscopic surgeries were performed by a neurosurgeon with much experience with thousands of neuroendoscopic surgeries. The level of the neurosurgeon's experience should also be considered in choice of operative approach.

We first analyzed the correlation between the Al-Mefty classification and prognosis. We found that the prognosis of patients with type I or II chordomas was significantly better than that for patients with type III tumors. Type I and II tumors are not very invasive, and radical resection can be achieved using a single skull base approach. Type III tumors invade surrounding structures extensively, which makes it difficult to achieve radical resection, and the residual chordoma recurs soon after. Thus, we consider this classification valuable to help surgeons decide on the appropriate surgery and predict prognosis.

Yang et al. analyzed 523 chordoma patients and showed that adjuvant radiotherapy may be beneficial in patients with positive surgical margins. ${ }^{29}$ Compared with conventional radiotherapy, proton beam radiotherapy could deliver higher radiation doses to the tumor region without increasing damage to surrounding structures. Several studies have reported that proton beam radiotherapy could improve local control rates and prognosis.${ }^{6,7}$ However, prospective studies are needed to define the role of postoperative radiotherapy, especially proton beam radiotherapy, in the management of clival chordoma. For our treatment team, if residual tumor is seen on postoperative imaging, patients are encouraged to receive radiotherapy.

PDGFR- $\beta$ is a cell surface tyrosine kinase receptor, which promotes cell transformation, cell migration, angiogenesis, and embryonic development. ${ }^{13,31}$ Higher expression of PDGFR- $\beta$ is associated with poorer prognosis in renal cell carcinoma, ${ }^{24}$ breast cancer, ${ }^{21}$ hepatocellular carcinoma,${ }^{5}$ and angiosarcoma, ${ }^{30}$ as well as other cancers. It has been reported that PDGFR- $\beta$ is highly expressed in chordomas. ${ }^{8,26}$ In our study, we first found that a higher expression level of PDGFR- $\beta$ was associated with poorer prognosis, indicating that PDGFR- $\beta$ could be added into pathological reports as a prognosis factor, which is more meaningful for patients who have undergone NTR.

In pancreatic cancer, mutant p53 increased PDGFR- $\beta$ expression through a cell-autonomous mechanism, which promoted metastasis. ${ }^{27}$ Blocking PDGFR- $\beta$ signaling by RNA interference prevented pancreatic cancer cell invasion in vitro and metastasis formation in vivo. Jechlinger et al. ${ }^{12}$ also reported that PDGFR- $\beta$ expression level correlated with invasive behavior in human breast cancer. The mTOR pathway plays an essential role in the regulation of tumor cell invasion and cancer metastasis. ${ }^{3}$ Inhibition of mTOR could diminish invasion and migration in gallbladder cancer ${ }^{33}$ and prostate cancer, ${ }^{4}$ as well as in other cancers. PDGFR- $\beta$ was a crucial upstream regulating factor in the pathway. Inhibition of PDGFR- $\beta$ could downregulate mTOR and pmTOR expression levels. Thus, we speculate 
that PDGFR- $\beta$ regulates chordoma cell invasion through the mTOR pathway.

\section{Conclusions}

Extent of resection, Al-Mefty classification, primary tumor, postoperative radiotherapy, and PDGFR- $\beta$ expression level are valuable prognostic factors for clival chordoma patients. PDGFR- $\beta$ could regulate invasion through the mTOR pathway in clival chordoma cells.

\section{Acknowledgments}

Chordoma cells (U-CH2) were donated by the Chordoma Foundation. This work was supported by the Research Special Fund for Public Welfare Industry of Health (201402008), the National High Technology Research and Development Program of China (863 Program, 2014AA020610), the National Natural Science Foundation of China (81271522), and the Innovation Foundation of Beijing Neurosurgical Institute (Youth-2014008).

\section{References}

1. Al-Mefty O, Borba LAB: Skull base chordomas: a management challenge. J Neurosurg 86:182-189, 1997

2. Berven LA, Willard FS, Crouch MF: Role of the $\mathrm{p} 70^{\mathrm{s} 6 \mathrm{~K}}$ pathway in regulating the actin cytoskeleton and cell migration. Exp Cell Res 296:183-195, 2004

3. Chen JS, Wang Q, Fu XH, Huang XH, Chen XL, Cao LQ, et al: Involvement of PI3K/PTEN/AKT/mTOR pathway in invasion and metastasis in hepatocellular carcinoma: association with MMP-9. Hepatol Res 39:177-186, 2009

4. Chen X, Cheng H, Pan T, Liu Y, Su Y, Ren C, et al: mTOR regulate EMT through RhoA and Rac1 pathway in prostate cancer. Mol Carcinog 54:1086-1095, 2015

5. Chu JS, Ge FJ, Zhang B, Wang Y, Silvestris N, Liu LJ, et al: Expression and prognostic value of VEGFR-2, PDGFR- $\beta$, and c-Met in advanced hepatocellular carcinoma. J Exp Clin Cancer Res 32:16, 2013

6. De Amorim Bernstein K, DeLaney T: Chordomas and chondrosarcomas - the role of radiation therapy. J Surg Oncol 114:564-569, 2016

7. Demizu Y, Mizumoto M, Onoe T, Nakamura N, Kikuchi Y, Shibata T, et al: Proton beam therapy for bone sarcomas of the skull base and spine: A retrospective nationwide multicenter study in Japan. Cancer Sci 108:972-977, 2017

8. Fasig JH, Dupont WD, LaFleur BJ, Olson SJ, Cates JM: Immunohistochemical analysis of receptor tyrosine kinase signal transduction activity in chordoma. Neuropathol Appl Neurobiol 34:95-104, 2008

9. Gui S, Zong X, Wang X, Li C, Zhao P, Cao L, et al: Classification and surgical approaches for transnasal endoscopic skull base chordoma resection: a 6-year experience with 161 cases. Neurosurg Rev 39:321-333, 2016

10. Heldin CH, Westermark B: Mechanism of action and in vivo role of platelet-derived growth factor. Physiol Rev 79:12831316, 1999

11. Hwang RF, Yokoi K, Bucana CD, Tsan R, Killion JJ, Evans $\mathrm{DB}$, et al: Inhibition of platelet-derived growth factor receptor phosphorylation by STI571 (Gleevec) reduces growth and metastasis of human pancreatic carcinoma in an orthotopic nude mouse model. Clin Cancer Res 9:6534-6544, 2003

12. Jechlinger M, Sommer A, Moriggl R, Seither P, Kraut N, Capodiecci P, et al: Autocrine PDGFR signaling promotes mammary cancer metastasis. J Clin Invest 116:1561-1570, 2006

13. Jones PS, Aghi MK, Muzikansky A, Shih HA, Barker FG II,
Curry WT Jr: Outcomes and patterns of care in adult skull base chordomas from the Surveillance, Epidemiology, and End Results (SEER) database. J Clin Neurosci 21:14901496, 2014

14. Kayani B, Sewell MD, Tan KA, Hanna SA, Williams R, Pollock R, et al: Prognostic factors in the operative management of sacral chordomas. World Neurosurg 84:1354-1361, 2015

15. Lavorato-Rocha AM, Anjos LG, Cunha IW, Vassallo J, Soares FA, Rocha RM: Immunohistochemical assessment of PTEN in vulvar cancer: best practices for tissue staining, evaluation, and clinical association. Methods 77-78:20-24, 2015

16. Liu L, Li F, Cardelli JA, Martin KA, Blenis J, Huang S: Rapamycin inhibits cell motility by suppression of mTOR-mediated S6K1 and 4E-BP1 pathways. Oncogene 25:7029-7040, 2006

17. Liu Q, Duan H, Luan J, Yagasaki K, Zhang G: Effects of theanine on growth of human lung cancer and leukemia cells as well as migration and invasion of human lung cancer cells. Cytotechnology 59:211-217, 2009

18. Maass T, Thieringer FR, Mann A, Longerich T, Schirmacher P, Strand D, et al: Liver specific overexpression of plateletderived growth factor-B accelerates liver cancer development in chemically induced liver carcinogenesis. Int J Cancer 128:1259-1268, 2011

19. McMaster ML, Goldstein AM, Bromley CM, Ishibe N, Parry DM: Chordoma: incidence and survival patterns in the United States, 1973-1995. Cancer Causes Control 12:1-11, 2001

20. Morgan TM, Koreckij TD, Corey E: Targeted therapy for advanced prostate cancer: inhibition of the PI3K/Akt/mTOR pathway. Curr Cancer Drug Targets 9:237-249, 2009

21. Paulsson J, Sjöblom T, Micke P, Pontén F, Landberg G, Heldin $\mathrm{CH}$, et al: Prognostic significance of stromal platelet-derived growth factor beta-receptor expression in human breast cancer. Am J Pathol 175:334-341, 2009

22. Samii A, Gerganov VM, Herold C, Hayashi N, Naka T, Mirzayan MJ, et al: Chordomas of the skull base: surgical management and outcome. J Neurosurg 107:319-324, 2007

23. Sen C, Triana AI, Berglind N, Godbold J, Shrivastava RK: Clival chordomas: clinical management, results, and complications in 71 patients. J Neurosurg 113:1059-1071, 2010

24. Shim M, Song C, Park S, Choi SK, Cho YM, Kim CS, et al: Prognostic significance of platelet-derived growth factor receptor- $\beta$ expression in localized clear cell renal cell carcinoma. J Cancer Res Clin Oncol 141:2213-2220, 2015

25. Steller EJ, Ritsma L, Raats DA, Hoogwater FJ, Emmink BL, Govaert KM, et al: The death receptor CD95 activates the cofilin pathway to stimulate tumour cell invasion. EMBO Rep 12:931-937, 2011

26. Tamborini E, Miselli F, Negri T, Lagonigro MS, Staurengo S, Dagrada GP, et al: Molecular and biochemical analyses of platelet-derived growth factor receptor (PDGFR) B, PDGFRA, and KIT receptors in chordomas. Clin Cancer Res 12:6920-6928, 2006

27. Weissmueller S, Manchado E, Saborowski M, Morris JP IV, Wagenblast E, Davis CA, et al: Mutant p53 drives pancreatic cancer metastasis through cell-autonomous PDGF receptor $\beta$ signaling. Cell 157:382-394, 2014

28. Wu Z, Zhang J, Zhang L, Jia G, Tang J, Wang L, et al: Prognostic factors for long-term outcome of patients with surgical resection of skull base chordomas-106 cases review in one institution. Neurosurg Rev 33:451-456, 2010

29. Yang L, Bai HX, Lee AM, Zou Y, Xiao B, Zhou J, et al: The role of radiotherapy in the treatment of spinal chordomas: an integrative analysis of 523 cases. Neuro Oncol 17:1419-1420, 2015

30. Yonemori K, Tsuta K, Ando M, Hirakawa A, Hatanaka Y, Matsuno Y, et al: Contrasting prognostic implications of platelet-derived growth factor receptor- $\beta$ and vascular en- 
dothelial growth factor receptor-2 in patients with angiosarcoma. Ann Surg Oncol 18:2841-2850, 2011

31. Yu J, Ustach C, Kim HR: Platelet-derived growth factor signaling and human cancer. J Biochem Mol Biol 36:49-59, 2003

32. Zhai Y, Bai J, Wang S, Du J, Wang J, Li C, et al: Differences in dural penetration of clival chordomas are associated with different prognosis and expression of platelet-derived growth factor receptor- $\beta$. World Neurosurg 98:288-295, 2017

33. Zong H, Yin B, Zhou H, Cai D, Ma B, Xiang Y: Inhibition of mTOR pathway attenuates migration and invasion of gallbladder cancer via EMT inhibition. Mol Biol Rep 41:45074512,2014

\section{Disclosures}

The authors report no conflict of interest concerning the materi- als or methods used in this study or the findings specified in this paper.

\section{Author Contributions}

Conception and design: Zhang, Zhai, Bai. Acquisition of data: Zhai, Wang, M Li. Analysis and interpretation of data: Zhai, Wang. Drafting the article: Zhai. Critically revising the article: Zhang, Zhai, Bai, Wang, Gao, C Li, Gui. Reviewed submitted version of manuscript: all authors. Approved the final version of the manuscript on behalf of all authors: Zhang. Statistical analysis: Zhai. Administrative/technical/material support: Zhang, Bai, Wang, Gao, C Li. Study supervision: Zhang, Bai, C Li, Gui.

\section{Correspondence}

Yazhuo Zhang: Beijing Neurosurgical Institute, Capital Medical University, Beijing, China. zyz2004520@yeah.net. 